Article

\title{
Optimal Bidding Strategy for Renewable Microgrid with Active Network Management
}

\author{
Seung Wan Kim ${ }^{1}$, Jip Kim ${ }^{2}$, Young Gyu Jin ${ }^{3, *}$ and Yong Tae Yoon ${ }^{1}$ \\ Received: 24 November 2015; Accepted: 11 January 2016; Published: 15 January 2016 \\ Academic Editor: G. J. M. (Gerard) Smit \\ 1 Department of Electrical and Computer Engineering, Seoul National University, 1 Gwanak-ro, \\ Gwanak-gu, Seoul 151-742, Korea; pc9873@snu.ac.kr (S.W.K.); ytyoon@snu.ac.kr (Y.T.Y.) \\ 2 Power System Research Division, Korea Electrical Engineering \& Science Research Institute, \\ Bldg. 130, 1 Gwanak-ro, Gwanak-gu, Seoul 151-742, Korea; jipkim@snu.ac.kr \\ 3 Center for Advanced Power \& Environmental Technology (APET), The University of Tokyo, 7-3-1 Hongo, \\ Bunkyo-ku, Tokyo 113-8656, Japan \\ * Correspondence: ygjin93@snu.ac.kr; Tel.: +82-2-880-9144; Fax: +82-2-885-4958
}

\begin{abstract}
Active Network Management (ANM) enables a microgrid to optimally dispatch the active/reactive power of its Renewable Distributed Generation (RDG) and Battery Energy Storage System (BESS) units in real time. Thus, a microgrid with high penetration of RDGs can handle their uncertainties and variabilities to achieve the stable operation using ANM. However, the actual power flow in the line connecting the main grid and microgrid may deviate significantly from the day-ahead bids if the bids are determined without consideration of the real-time adjustment through ANM, which will lead to a substantial imbalance cost. Therefore, this study proposes a formulation for obtaining an optimal bidding which reflects the change of power flow in the connecting line by real-time adjustment using ANM. The proposed formulation maximizes the expected profit of the microgrid considering various network and physical constraints. The effectiveness of the proposed bidding strategy is verified through the simulations with a 33-bus test microgrid. The simulation results show that the proposed bidding strategy improves the expected operating profit by reducing the imbalance cost to a greater degree compared to the basic bidding strategy without consideration of ANM.
\end{abstract}

Keywords: active network management; market participation of microgrid; microgrid operation; renewable microgrid

\section{Introduction}

Microgrids (MGs) are medium or low voltage distribution networks that comprise distributed generations, electrical loads, and energy storage systems [1,2]. A Microgrid Operator (MGO) aims to achieve stable and efficient operation of the MG through the proper use of the available resources $[3,4]$. In particular, in the grid-connected mode, a MGO can participate in the electricity market to buy or sell energy to maintain the balance between supply and demand of the MG in a most economical way $[5,6]$. Recently, much research has been conducted on establishing an optimal bidding strategy for the MGO in charge of operating MGs with high penetration of Renewable Distributed Generation (RDG) systems, the so-called renewable MGs [7-11]. Stochastic approaches have been commonly used in the relevant research [7-11] to obtain an optimal bidding strategy for renewable MGs, because RDGs produce significantly uncertain and variable output compared to conventional generators. Optimal bidding and operation strategies are derived in [7-11] to minimize expected operating costs through a stochastic approach considering the uncertainties in the outputs of Wind Turbines (WTs) or Photovoltaics (PVs), and demand. In particular, the previous approaches in [7-9] focus on the scheduling of dispatchable 
resources in the MG rather than strategic bidding of the MGO in the electricity market. Further in $[10,11]$, the MGO considers the imbalance cost caused by the difference between the submitted bid and the actual power flow in the line connecting the main grid and MG. In [11], a tradeoff between maximizing the expected operating profit of the MG and reducing the risk of low expected operating profit in unfavorable scenarios is considered to develop a bidding strategy for the MGO. Furthermore, in [11] it is shown that the MGO can increase its profit by utilizing demand response as an additional controllable resource.

The bidding strategies proposed in [7-11] do not however consider network conditions and physical constraints, such as lower and upper voltage limits for each bus and line capacities. Furthermore, although the reactive power balance in the MG is as important as the active power balance for stable operation of a MG network, none of previous approaches described in [7-11] consider the reactive power demands in their formulation of an optimal bidding strategy. For these reasons, there is a high risk of violating the physical constraints in real-time operation. As a solution for resolving this problem, the MGO can curtail the active power outputs of RDGs, adjust their power factors, or schedule active/reactive powers of the Battery Energy Storage Systems (BESSs) in real-time operation.

The above real-time adjustments of the MGO can be realized through Active Network Management (ANM) [12-15]. Owing to the real-time adjustments using ANM, the power flow in the line connecting the main grid and MG can deviate significantly from the submitted bid, which is obtained according to the bidding strategies proposed in [10,11]. For this reason, the MGO is likely to be charged for a significant imbalance cost. Therefore, this study proposes an optimal bidding strategy that enables the MGO to consider the change of the power flow on the connected line by real-time adjustments using ANM, through a scenario-based stochastic optimization method. By using the proposed bidding strategy, the imbalance cost can be reduced and the expected operating profit can be improved.

The remainder of the paper is organized as follows: in Section 2, the assumptions for the market participation of the MGO are summarized. Section 3 describes the basic bidding strategy of the MGO without consideration of ANM. Section 4 introduces the features of ANM in MG operation, and proposes the optimization approach to achieve an optimal bidding strategy considering ANM for day-ahead bidding. Section 5 presents the limitations of the basic bidding strategy described in Section 3 through simulation, and verifies the improvement in the daily expected operating profit when the proposed bidding strategy is used. Finally, Section 6 contains the conclusions of this study.

\section{Microgrid Operators as Market Participant}

In this study, it is assumed that a MGO is allowed to participate in the day-ahead energy market and submit bids as either a supplier or customer, according to the expected direction of the power flow in the line connecting the main grid and MG. In order to forecast the quantity and direction of power flow in the connecting line, it is necessary for the MGO to know forecasted values for electricity demands of buses and RDG outputs in the MG network. Various artificial intelligence-based forecasting techniques can be effectively used to obtain more accurate forecasting data in a MG environment $[16,17]$.

However, the MGO in charge of operating a renewable MG is unwillingly to violate the submitted bid in the day-ahead bidding because of the large uncertainties in the forecasted outputs of RDGs. The difference between the submitted bid in the day-ahead bidding and the actual power flow in the line connecting in the real-time operation is defined as an imbalance [18]. In order to keep the main grid reliable and stable in the presence of this imbalance, the System Operator (SO) of the main grid has to pay extra costs to secure reserves, or prepare flexible resources. Therefore, the SO penalizes the MGO who causes the imbalance. Figure 1 shows the principle of the charging imbalance cost for market participants. If power flow in the connecting line is greater than the upper limit of the tolerance band, the violation is charged at the penalty price for under-scheduling imbalance. Under-scheduling imbalance causes a power shortage in the main grid. Moreover, if the power flow on the connecting 
line is less than the lower limit of the tolerance band, the violation is charged at the penalty price for over-scheduling imbalance. Over-scheduling imbalance causes a surplus of power in the main grid. However, no imbalance cost is charged if the power flow in the connecting line is within the tolerance band of the submitted bid. Table 1 summarizes the market rules for the calculation of imbalance cost used in the previous researches $[10,11]$ as well as in the electricity market [19]. As shown in Table 1, $[10,11]$ use a simplified rule to calculate the imbalance cost. They do not consider the tolerance band is which the penalty price is not charged. On the contrary, the tolerance band is considered in the practical market rule for imbalance cost calculation in [19]. Meanwhile, the amount of energy exchanged with the MGO is very small compared to the whole amount traded in the market. Thus, in this study it is assumed that the MGO is a price-taker that does not affect market price.

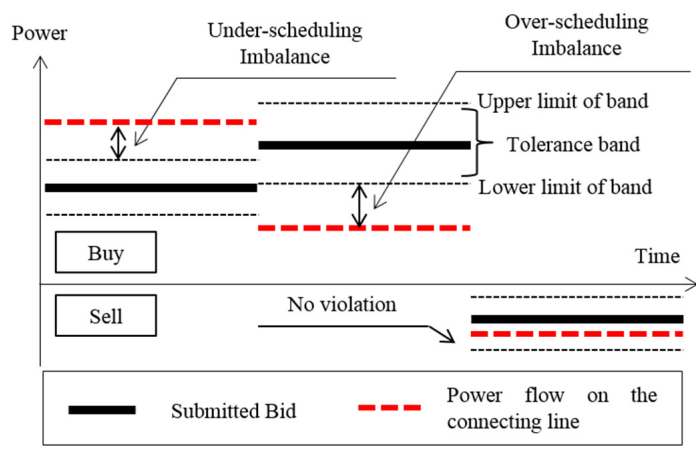

Figure 1. Conceptual depiction of imbalance cost.

Table 1. Market rules for imbalance cost.

\begin{tabular}{ccccc}
\hline \multirow{2}{*}{ Reference } & Tolerance Band & Threshold Level & \multicolumn{2}{c}{ Penalty Price Factor } \\
\cline { 3 - 5 } & & Noner-Scheduling & $\begin{array}{c}\text { Under-Scheduling } \\
\text { Imbalance }\end{array}$ \\
\hline Shi et al. $[10]$ & None & None & $20 \%$ of MP & $15 \%$ of MP \\
Nguyen et al. [11] & None & $>5 \%$ & $\begin{array}{c}\text { Peak price of } 24 \mathrm{~h} \\
25 \% \text { of MP }\end{array}$ & $\begin{array}{c}\text { Peak price of } 24 \mathrm{~h} \\
25 \% \text { of } \mathrm{MP}\end{array}$ \\
CAISO [17] & $\pm 5 \%$ of bid & $>10 \%$ & $50 \%$ of MP & $100 \%$ of MP \\
\hline
\end{tabular}

\section{Basic Bidding Strategy Without Active Network Management}

This section describes the basic bidding strategies based on the previous studies without considering ANM [10,11]. In the basic approach, the MGO determines optimal bids for the next $24 \mathrm{~h}$ in the day-ahead market through scenario-based stochastic optimization in order to maximize the expected operating profit, represented as Equation (1), subject to the constraint given by Equations (2)-(6):

$$
\begin{gathered}
\max \sum p^{s} \cdot P R^{s} \\
=\max \sum p^{s} \cdot\left[R^{s}-\left(C_{G}^{s}+C_{B W}^{s}+C_{E X}^{s}+C_{I M B}^{s}\right)\right]
\end{gathered}
$$

subject to:

$$
\begin{gathered}
-\bar{S}_{C O N} \leqslant P_{C O N, t}^{s} \leqslant \bar{S}_{C O N} \\
-P_{B E S S, i}^{M A X} \leqslant P_{B E S S, i, t}^{s} \leqslant P_{B E S S, i}^{M A X} \\
S o C_{i}^{M I N} \leqslant S o C_{i, t}^{s} \leqslant S o C_{i}^{M A X} \\
S o C_{N, i}^{s}=S o C_{0, i}^{s}-\sum_{t=1}^{N} P_{B E S S, i, t}^{s} \cdot \Delta t \\
P_{C O N, t}^{s}+\sum_{i \in B} \hat{P}_{G, i, t}^{s}+\sum_{i \in B} P_{B E S S, i, t}^{s}=\sum_{i \in B} \hat{P}_{D, i, t}^{s}
\end{gathered}
$$


The functions of revenue and other costs in Equation (1) are explained in Section 4 below. The power flow on the connecting line $P_{\mathrm{CON}, t}^{s}$ should not exceed the capacity of the corresponding line as in Equation (2). Further, the charging/discharging power $P_{B E S S, i, t}^{s}$ is limited by the capacity of the power conditioning system as formulated in Equation (3). The state-of-charge $S o C_{i, t}^{\mathrm{s}}$ of the $i$-th BESS should remain within the range of Equation (4) to avoid damage to the battery due to deep discharging or overcharging, and $S o C_{i, t}^{\mathrm{s}}$ can be calculated using the equality constraint in Equation (5). For simplicity, the charging/discharging efficiencies of BESSs are assumed to be $100 \%$ in this study. Finally, the constraint on the active power balance of the supply and demand in the MG is represented in Equation (6).

\section{Proposed Bidding Strategy with Active Network Management}

\subsection{Features of Active Network Management in Microgrid Operation}

A renewable $M G$ suffers from problems because of the intermittent outputs of RDGs. For example, an under-voltage problem may occur at certain buses in the MG if there is no wind or sunshine at certain times. On the other hand, an over-voltage problem may occur at certain buses in the MG, if RDGs generate excessive output according to the weather conditions at certain times. ANM can be a solution to these operational problems. ANM is a smart-grid technology that enables a MGO to measure the state of each bus, such as the voltage and angle, in real-time; calculate the optimal dispatch of resources considering the network conditions and physical constraints; and then deliver the dispatched signals to resources through a communication infrastructure [12-15]. Therefore, a MGO with ANM is able to stably and efficiently operate a renewable MG by optimally curtailing the outputs of RDGs and loads and scheduling the active/reactive powers of BESSs without violating physical constraints.

\subsection{Formulation of Optimal Bidding Strategy with Active Network Management}

Similar to the basic bidding strategy described in Section 3, the proposed bidding strategy aims to maximize the daily expected operating profit. However, the proposed bidding strategy is significantly different from the basic bidding strategy in that additional control variables, constraints and extra cost factors related to ANM are included in its formulation.

\subsubsection{Objective function}

The objective function of the optimization problem for an optimal bidding strategy with ANM is as follows:

$$
=\max \sum p^{s} \cdot\left[\begin{array}{r}
\max \sum p^{s} \cdot P R^{s} \\
\mathrm{R}^{s}-\left(C_{L C U R T}^{s}+C_{G}^{s}+C_{G C U R T}^{s}+C_{L o s s, s}\right. \\
\left.+C_{E X, s}+C_{I M B, s}+C_{B W, s}\right)
\end{array}\right]
$$

A MGO with ANM is able to adjust the forecasted demand $\hat{P}_{D, i, t}^{s}$ to the dispatched load $P_{L, i, t}^{s}$ during the real-time operation. Therefore, unlike the basic bidding strategy that uses $\hat{P}_{D, i, t}^{s}$ the revenue in the proposed bidding strategy can be calculated with $P_{L, i, t}^{s}$ as:

$$
\mathrm{R}^{S}=\sum_{t \in T} \rho_{t} \sum_{i \in B} P_{L, i, t}^{s}
$$

The MGO needs to pay an incentive or a compensation for load curtailment to consumers who have contracted curtailable loads. This study assumes that all of the loads in the MG are curtailable. The cost of load curtailment can be modeled as follows:

$$
C_{L C U R T}^{s}=\sum_{t \in T} \gamma_{L C U R T} \lambda_{t} \sum_{i \in B}\left(\hat{P}_{D, i, t}^{s}-P_{L, i, t}^{s}\right)
$$


where the incentive price for load curtailment can be set by multiplying $\lambda_{t}$ by $\gamma_{L C U R T}$. The form of the compensation price $\gamma_{L C U R T} \lambda_{t}$ varies according to the policy, tariff, or contracts with consumers.

The generation cost paid for RDGs should be calculated with the dispatched active power output $P_{G, i, t}^{s}$ rather than the forecasted output $\hat{P}_{G, i, t}^{s}$ because the MGO with ANM is able to curtail the outputs of RDGs. In this study, it is assumed that the curtailed quantity $\hat{P}_{G, i, t}^{s}-P_{G, i, t}^{s}$ is compensated at the compensation price $\gamma_{G C U R T} \lambda_{t}$, where $\gamma_{G C U R T}$ can be determined according to the various policies of the electricity company, or regulator. The costs incurred from these two factors are represented as:

$$
\begin{gathered}
C_{G}^{s}=\sum_{t \in T} \sum_{i \in B} \lambda_{t} P_{G, i, t}^{s} \\
C_{G C U R T}^{s}=\sum_{t \in T} \gamma_{G C U R T} \lambda_{t} \sum_{i \in B}\left(\hat{P}_{G, i, t}^{s}-P_{G, i, t}^{s}\right)
\end{gathered}
$$

Because the lines of the distribution network in the MG have larger R/X ratios than a high voltage transmission line, it is not desirable to neglect the cost of the line loss due to the line resistance. In particular, the MGO with ANM is able to calculate this cost by monitoring state information such as the voltage and angle, at each bus. The cost of the line loss can be calculated at $\lambda_{t}$ as follows:

$$
\begin{gathered}
C_{\text {LOSS }}^{s}=\sum_{t \in T} \lambda_{t} f_{\text {LOSS }, t}^{s} \\
f_{\text {LOSS }, t}^{s}=\sum_{i=1}^{n} \sum_{j=1}^{n} G_{i j}\left(\left|V_{i, t}^{s}\right|^{2}+\left|V_{j, t}^{s}\right|^{2}+2\left|V_{i, t}^{s}\right|\left|V_{j, t}^{s}\right| \cos \left(\delta_{i, t}^{s}-\delta_{j, t}^{s}\right)\right)
\end{gathered}
$$

The MGO participates in the day-ahead energy market to buy or sell energy for balancing the supply and demand of the MG. The exchange $\operatorname{cost} C_{E X}^{s}$ charged for line flow $P_{C O N, t}^{s}$ which is delivered through the line connecting the main grid and $M G$, can be represented as follows:

$$
C_{E X}^{s}=\sum_{t \in T} \lambda_{t} P_{\mathrm{CON}, t}^{s}
$$

Note that $P_{\mathrm{CON}, t}^{s}$ is positive when the MG draws power from the main grid, and negative when the MG provides power to the main grid.

In this study, the imbalance cost is modeled according to the principle described in Figure 1 as follows:

$$
\begin{gathered}
C_{I M B}^{s}=\sum_{t \in T} \mu_{U N D E R} \cdot \lambda_{t} \cdot I\left(P_{C O N, t}^{s}-(1+b) \cdot P_{B I D, t}\right) \\
+\sum_{t \in T} \mu_{O V E R} \cdot \lambda_{t} \cdot I\left((1-b) \cdot P_{B I D, t}-P_{C O N, t}^{s}\right) \\
I(x)=\left\{\begin{array}{lll}
x & \text { if } & x>0 \\
0 & \text { if } & x \leqslant 0
\end{array}\right.
\end{gathered}
$$

Further, it is assumed that the MGO has BESS(s) for economic and stable MG operation. Accordingly, the MGO has to consider the cost of battery wear in order to avoid excessive operation of the BESS. The cost of battery wear can be adopted from [20] as:

$$
C_{B W}^{S}=\sum_{t \in T} \sum_{i \in B}\left(\alpha_{B} \cdot P_{B E S S, i, t}^{S}-\beta_{B} \cdot P_{B E S S, i, t}^{S} \cdot P_{B E S S, i, t+1}^{S}\right)
$$

\subsubsection{Load constraints}

By considering the load curtailment capability of ANM, the ranges of the dispatched active/reactive loads can be represented as:

$$
0 \leqslant P_{L, i, t}^{s} \leqslant \hat{P}_{D, i, t}^{s} \quad 0 \leqslant Q_{L, i, t}^{s} \leqslant \hat{Q}_{D, i, t}^{s}
$$


However, the load power factor of each bus should be maintained even in a curtailed schedule because the power factor reflects the characteristics of the load [21]. Hence, it is necessary to consider the constraints on the power factors of the loads as follows:

$$
\frac{Q_{L, i, t}^{s}}{P_{L, i, t}^{s}}=\frac{\hat{Q}_{D, i, t}^{s}}{\hat{P}_{D, i, t}^{s}}
$$

\subsubsection{Generation constraints}

In general, the maximum output of a conventional generator is specified at its nominal capacity. On the contrary, the maximum outputs of RDGs, such as WTs and PV systems, depend on the weather conditions at the time and are less than the given nominal capacity. Thus, the MGO can adjust the output of an RDG within a certain range given by:

$$
0 \leqslant P_{G, i, t}^{s} \leqslant \hat{P}_{G, i, t}^{s} \leqslant P_{C A P, i}
$$

The active/reactive powers can be freely adjusted within the given capability curve, because an RDG is typically an inverter-based machine. For example, Figure 2a shows the capability curve for both WT and PV with the power factor that ranges from the 0.9 lagging to the 0.9 leading. This capability curve in Figure 2a can be represented as inequality constraints as follows:

$$
\begin{gathered}
P_{G, i, t}^{s}{ }^{2}+Q_{G, i, t}^{s} \leqslant \hat{P}_{G, i, t}^{s}{ }^{2} \\
-P_{G, i, t}^{s} \tan \left(\cos ^{-1}\left(P F R_{G}\right)\right) \leqslant Q_{G, i, t}^{s} \leqslant P_{G, i, t}^{s} \tan \left(\cos ^{-1}\left(P F R_{G}\right)\right)
\end{gathered}
$$

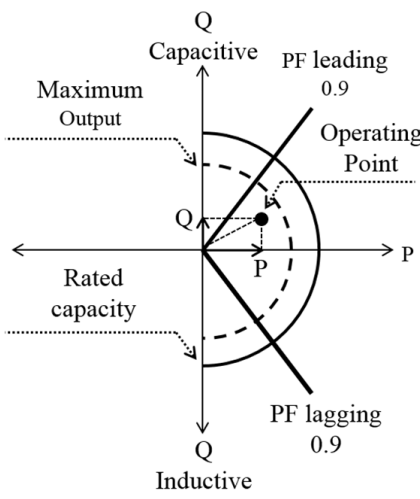

(a)

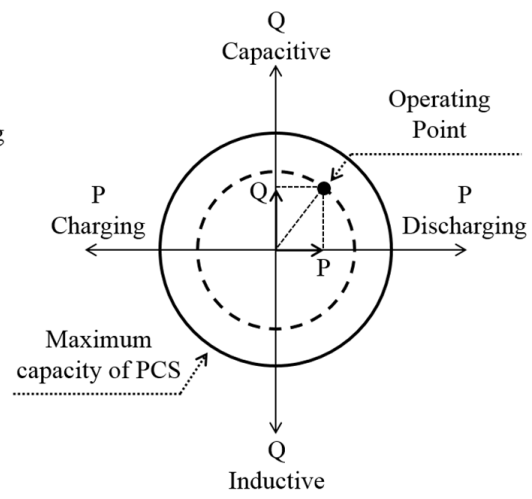

(b)

Figure 2. Capability curves of (a) a RDG and (b) a BESS.

\subsubsection{BESS constraints}

A BESS consists of a battery cell, which charges/discharges a direct current, and a power conditioning system, which converts a direct current into an alternating current:

$$
\begin{gathered}
P_{B E S S, i, t}^{s}{ }^{2}+Q_{B E S S, i, t}^{s} \leqslant P_{B E S S, i}^{M A X}{ }^{2} \\
S o C_{i}^{M I N} \leqslant S o C_{i, t}^{s} \leqslant S o C_{i}^{M A X} \\
S o C_{N, i}^{s}=S o C_{0, i}^{s}-\sum_{t=1}^{N} P_{B E S S, i, t}^{s} \cdot \Delta t
\end{gathered}
$$


The power conditioning system of the BESS can adjust the power factor of the output of the BESS within the four quadrants of the capability curve in Figure $2 b$ [22]. The capability curve can be modeled by Equation (23). Equations (24) and (25) are the same as those for the basic bidding strategy in Section 3.

\subsubsection{Power flow constraints}

The MGO with ANM is able to obtain state information, such as the voltage and angle, at each bus. Hence, the power flow related constraints can be included in the optimization:

$$
\begin{gathered}
P_{C O N, t}^{s}+P_{G, i, t}^{s}+P_{B E S S, i, t}^{s}-P_{L, i, t}^{s}-P_{F, l, t}^{s}=0, i=1 \\
Q_{C O N, t}^{s}+Q_{G, i, t}^{s}+Q_{B E S S, i, t}^{s}-Q_{L, i, t}^{s}-Q_{F, l, t}^{s}=0, i=1 \\
P_{G, i, t}^{s}+P_{B E S S, i, t}^{s}-P_{L, i, t}^{s}-P_{F, l, t}^{s}=0, i \neq 1 \\
Q_{G, i, t}^{s}+Q_{B E S S, i, t}^{s}-Q_{L, i, t}^{s}-Q_{F, l, t}^{s}=0, i \neq 1
\end{gathered}
$$

where:

$$
\begin{aligned}
& P_{F, l, t}^{s}=\left|V_{i, t}^{s}\right| \sum_{j}\left|V_{j, t}^{s}\right|\left[G_{i j} \cos \left(\delta_{i, t}^{s}-\delta_{j, t}^{s}\right)+B_{i j} \sin \left(\delta_{i, t}^{s}-\delta_{j, t}^{s}\right)\right] \\
& Q_{F, l, t}^{s}=\left|V_{i, t}^{s}\right| \sum_{j}\left|V_{j, t}^{s}\right|\left[G_{i j} \sin \left(\delta_{i, t}^{s}-\delta_{j, t}^{s}\right)-B_{i j} \cos \left(\delta_{i, t}^{s}-\delta_{j, t}^{s}\right)\right]
\end{aligned}
$$

At Bus 1, which is linked to the line connecting the main grid and MG, the active/reactive powers should be satisfied for the power balancing Equation (26). Similarly, the active/reactive power balancing Equation (27) must be satisfied at all buses except the first bus. The constraints for Bus 1 in Equation (26) additionally include $P_{C O N, t}^{s}$ and $Q_{C O N, t}^{s} . P_{F, i, s}^{t}$ and $Q_{F, i, s}^{t}$ can be represented as in Equation (28). The power flows in the distribution lines and the connecting line should not exceed their respective line capacities, which can be represented as follows:

$$
\begin{gathered}
P_{F, l, t}^{s}{ }^{2}+Q_{F, l, t}^{s}{ }^{2} \leqslant \bar{S}_{\text {LINE, }{ }^{2}}{ }^{2}{ }^{2} \leqslant \bar{S}_{\mathrm{CON}}{ }^{2} \\
P_{\mathrm{CON}, t}^{s}+Q_{\mathrm{CON}, t}^{s}{ }^{2}
\end{gathered}
$$

Additionally, the power flow in the connecting line should be maintained within the power factor range predetermined by the contract with the $\mathrm{SO}$ :

$$
\left(Q_{\mathrm{CON}, t}^{s}\right)^{2} \leqslant\left(P_{\mathrm{CON}, t}^{s} \tan \left(\cos ^{-1}\left(\text { PFR }_{\mathrm{CON}}\right)\right)\right)^{2}
$$

Finally, the voltages of all buses in the network should be within the regulation range as follows:

$$
V_{L L} \leqslant\left|V_{i, t}^{S}\right| \leqslant V_{U L}
$$

\section{Simulation}

\subsection{Simulation Settings}

The performance of the proposed bidding strategy is verified through simulations with a renewable MG based on the IEEE 33-bus distribution network in [23]. The structure of the MG is shown in Figure 3. Detailed data for the branches are taken from [23]. It is assumed that five WTs, five PVs, and five BESSs are installed in the MG. The capacities of the WTs and PVs are selected to render the MG self-sufficient, such that the sum of the nominal capacities is greater than the daily peak load of the MG. Further, the maximum charging/discharging power in MVA of a BESS is set to half of its capacity in MWh. The specific data related to the WTs, PVs, and BESSs are listed in Table 2. The base values of power and voltage are set to 1 MVA and $12.66 \mathrm{kV}$, respectively. 
The forecasted hourly demands in the MG as presented in Figure 4 are taken from the California Independent System Operator (CAISO) for 2 July 2015 [24]. The active/reactive power demand of each bus is assumed to be distributed according to the data in [23]. It is assumed that the ratio of the forecasted hourly output of each WT to its capacity is the same for all WTs, and the values for this ratio are taken from [25]. The same assumption is applied to the forecasted hourly output of each $\mathrm{PV}$, and the ratio from [7] is used for the all PVs. The forecasted hourly outputs during $24 \mathrm{~h}$ for WTs and PVs are presented in Figure 5. The forecasting errors for the demand, the WT output, and the PV output in each hour are all assumed to be normally distributed with zero mean. The standard deviations are set to $2 \%$ of the forecasted demand [26], $10 \%$ of the forecasted WT output [25], and $20 \%$ of the PV output [10], respectively. On the basis of these forecasting error models, 1000 scenarios are generated using Monte Carlo simulations, and 10 representative scenarios are selected from them using the scenario-reduction method in [10].

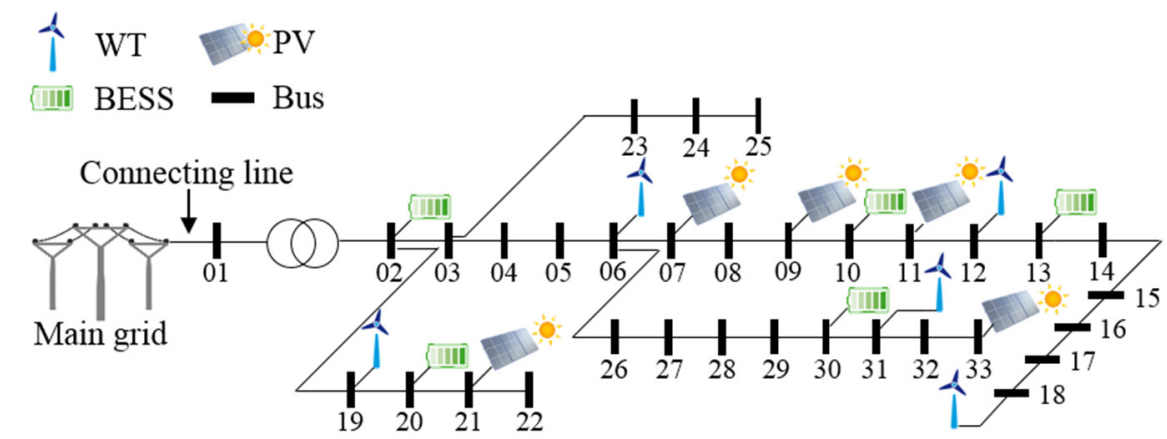

Figure 3. Renewable microgrid based on the IEEE 33-bus distribution network.

Table 2. Capacities and locations of WTs, PVs, and BESSs.

\begin{tabular}{ccccccc}
\hline Resource types & Properties & \multicolumn{7}{c}{ Values } \\
\hline \multirow{2}{*}{ WT } & Location & Bus 6 & Bus 12 & Bus 18 & Bus 19 & Bus 31 \\
& Capacity (MVA) & 1.2 & 0.6 & 0.6 & 0.96 & 1.2 \\
\hline \multirow{2}{*}{ PV } & Location & Bus 7 & Bus 9 & Bus 11 & Bus 21 & Bus 33 \\
& Capacity (MVA) & 0.24 & 0.36 & 0.36 & 0.36 & 0.6 \\
\hline \multirow{3}{*}{ BESS } & Location & Bus 2 & Bus 10 & Bus 13 & Bus 20 & Bus 30 \\
& Max. Power (MVA) & 0.18 & 0.06 & 0.06 & 0.18 & 0.30 \\
& Capacity (MWh) & 0.36 & 0.12 & 0.12 & 0.36 & 0.60 \\
\hline
\end{tabular}

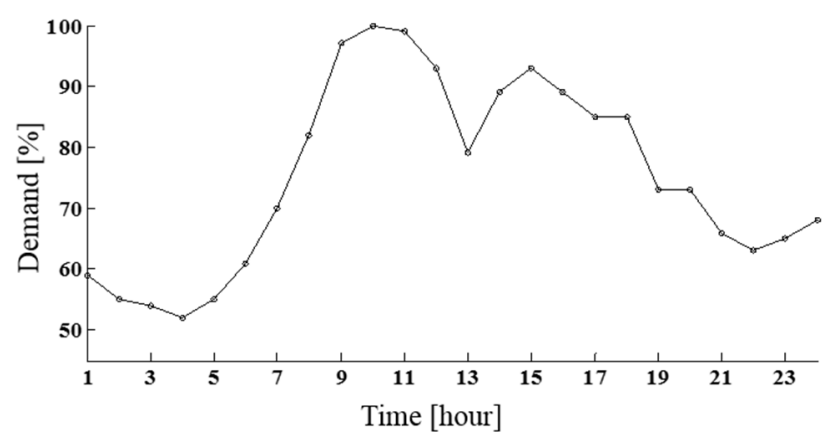

Figure 4. Forecasted hourly demand for $24 \mathrm{~h} \mathrm{[24].}$ 


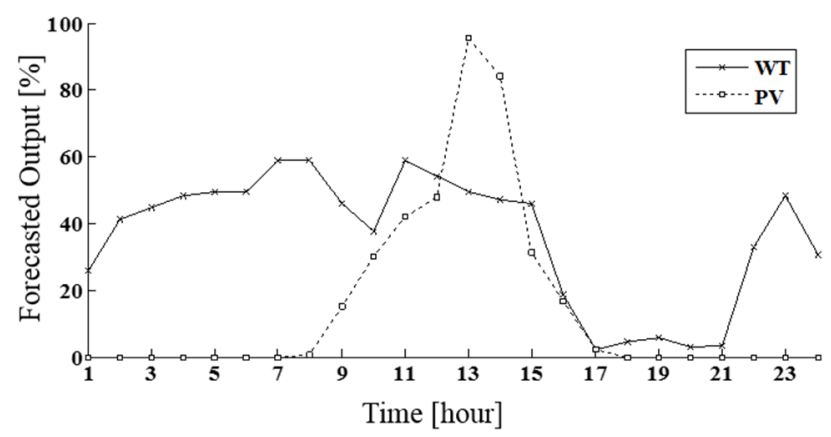

Figure 5. Forecasted hourly output of WT and PV normalized to their nominal capacity.

The day-ahead market prices of CAISO for 2 July 2015 [20] are used for $\lambda_{t}$, which are presented in Figure 6. For the retail price $\rho_{\mathrm{t}}$, the Time-of-Use (TOU) pricing scheme of the Pacific Gas and Electric Company (PG\&E, Sacramento, CA, USA) with off-peak (\$38.71/MWh), mid-peak (\$77.42/MWh), and on-peak ( $\$ 190.35 / \mathrm{MWh})$ prices is used [27].

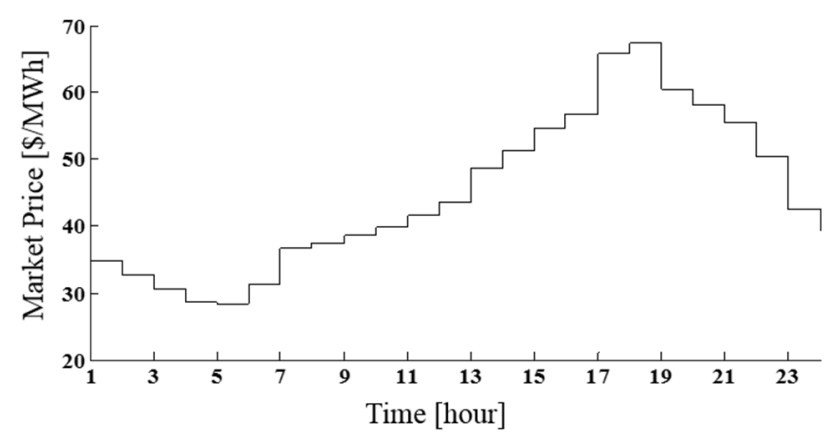

Figure 6. Day-ahead market price in CAISO on 2 July 2015.

Two simulation cases are designed to examine the effect of the penalty price by applying different market rule as listed in Table 3. Case I and Case II have the same tolerance band, but the penalty price factor for Case II is set to 1.5 times larger than that for Case I.

Table 3. Assumed market rules for imbalance cost in simulation.

\begin{tabular}{cccc}
\hline \multirow{2}{*}{ Simulation Case } & \multirow{2}{*}{ Tolerance Band } & \multicolumn{2}{c}{ Penalty Price Factor } \\
\cline { 3 - 4 } & & Over-Scheduling & Under-Scheduling \\
\hline Case I & \multirow{2}{*}{ $\pm 5 \%$} & $50 \%$ & $50 \%$ \\
Case II & $75 \%$ & $75 \%$ \\
\hline
\end{tabular}

The values of the other parameters in Equations (7)-(31) are listed in Table 4. The optimization problems for both the basic and proposed bidding strategies are solved using GAMS/BONMIN solver on a PC with a $2.30 \mathrm{GHz}$ Intel ${ }^{\circledR}$ Core i5 CPU, 8 GB RAM, and a 64-bit Windows ${ }^{\circledR} 7$ operating system. The relative gap of GAMS/BONMIN Solver is set to $0.1 \%$. 
Table 4. Values of other parameters in Equations (7)-(31).

\begin{tabular}{cc}
\hline Generation curtailment compensation factor $\gamma_{G C U R T}$ & $80 \%$ \\
Load curtailment compensation factor $\gamma_{L C U R T}$ & $300 \%$ \\
Fast charging/cycling cost parameters $\alpha_{B}, \beta_{B}$ & $1 / 0.75$ \\
Minimum/maximum state-of-charge of the BESS & $20 \% / 95 \%$ \\
$\Delta t$ & $1 \mathrm{~h}$ \\
Predetermined power factor range of RDGs & 0.9 Lagging-0.9 Leading \\
Predetermined power factor range of the line & 0.95 Lagging-0.95 Leading \\
connecting the main grid and $M G$ & 0.95 p.u. $/ 1.05$ p.u. \\
Lower/upper voltage limit $V_{L L}, V_{U L}$ & \\
\hline
\end{tabular}

\subsection{Simulation Results}

If ANM is not used in real-time operation of renewable MG, the RDGs would simply generate the maximum outputs determined by the weather conditions with a fixed unity power factor. In addition, the BESSs would operate according to the active power schedules without considering the network conditions. Then, the voltages at some buses in the MG may not be maintained between the lower and upper voltage limits, and the power flows in some distribution lines may exceed their respective line capacities. Indeed, with the given simulation settings, the under-voltage problem is observed in Buses $10-18$ and $28-33$ at $t=17$ for one representative scenario in Figure $7 \mathrm{a}$. This is because the reactive power supplies from the RDGs or BESSs are not at all scheduled to meet the reactive power demand in the MG, when the basic bidding strategy without ANM being applied. On the other hand, the over-voltage problem is not observed for all representative scenarios, because the output patterns of RDGs are not sufficiently excessive to cause the voltage values of some buses to exceed the upper-voltage limit with the given simulation settings. Further, the power flow of each distribution line does not violate the line capacities with the given simulation settings. Because the line capacities of the test MG are already sufficiently designed to endure the power flow required to meet the demand, the power flow can be considerably reduced by the outputs of the RDGs. Although the under-voltage problem is only observed with the given simulation settings, the over-voltage problem and the violation of line capacity can be observed in other configurations, particularly with large outputs of the RDGs, less demand, or smaller line capacities.

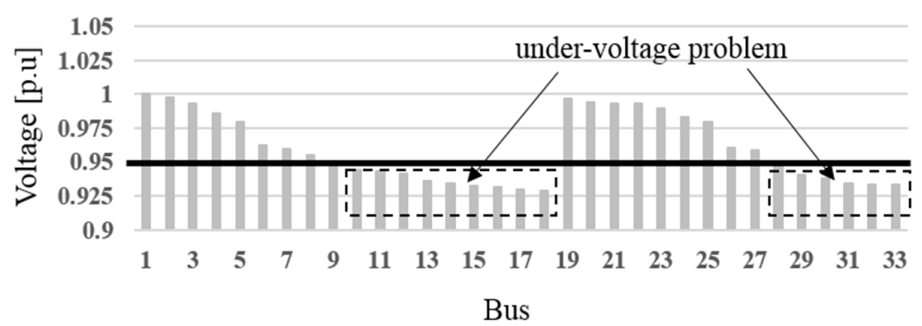

(a)

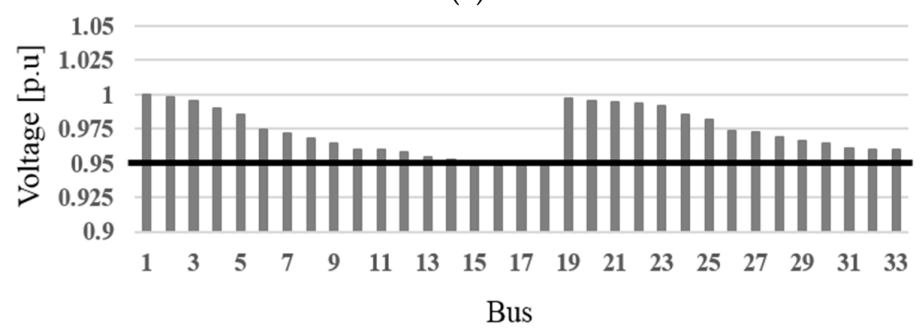

(b)

Figure 7. Simulated voltage profiles of the test $M G$ at $t=17$ for one representative scenario (a) without the real-time operation with ANM and (b) with the real-time operation with ANM for the basic bidding strategy. 
In order to resolve the under-voltage problem as shown in Figure 7a, the MGO needs real-time adjustments with ANM such as a curtailment of the outputs of the RDGs and loads and an optimal schedule the active/reactive powers of BESSs. Therefore, it is assumed that real-time adjustments with ANM is also applied, even if the basic bidding strategy is used. As result of real-time adjustments using ANM, the voltages of all buses in the MG can be maintained within the voltage limits as shown in Figure $7 \mathrm{~b}$. However, the power flow in the connecting line significantly deviate from the submitted bid based on the basic bidding strategy. In other words, the basic bidding strategy can cause a large imbalance cost due to the modified power flow in the connecting line while addressing the physical constraints. On the contrary, the proposed bidding strategy considers such as the ANM capabilities when determining day-ahead bids. Therefore, the deviation from the submitted bid and the resulting imbalance cost can be small.

The simulated daily expected operating revenue, costs and profit for the basic and proposed bidding strategies are presented in Table 5 . From Table 5, the daily expected operating profit of the proposed bidding strategy is improved compared to the basic bidding strategy by $1.5 \%(\$ 41.01)$ for Case I and 2.34\% (\$63.48) for Case II. This is because the basic bidding strategy causes relatively larger imbalance costs than the proposed bidding strategy. These larger imbalance costs are due to the way in which the MGO determines the bids in the basic bidding strategy without consideration of the real-time adjustments using ANM. Figure 8 shows the hourly imbalance normalized to the bid for each hour in the basic and proposed strategies in both cases. It can be clearly seen that the proposed bidding strategy significantly reduces the imbalance. Consequently, as presented in Table 5, the MGO can increase the expected operating profit using the proposed bidding strategy considering ANM.

Table 5. Expected Operating Revenue, Costs and Profit of Two Bidding Strategies for the Simulation Cases.

\begin{tabular}{lccccccc}
\hline \multirow{2}{*}{ Simulation Case } & \multicolumn{3}{c}{ Case I } & \multicolumn{3}{c}{ Case II } \\
\cline { 2 - 7 } & Revenue & Basic & Proposed & Difference & Basic & Proposed & Difference \\
\hline \multirow{4}{*}{ Costs } & 6484.14 & 6483.90 & -0.24 & 6483.62 & 6482.64 & -0.98 \\
\cline { 2 - 8 } & Exchange cost & 1562.30 & 1562.90 & 0.60 & 1563.24 & 1571.00 & 7.77 \\
& Imbalance cost & 54.04 & 13.10 & -40.94 & 78.44 & 9.55 & -68.90 \\
& Loss cost & 66.55 & 65.68 & -0.86 & 67.26 & 64.91 & -2.35 \\
& Battery wear cost & 0.18 & 0.18 & 0.00 & 0.20 & 0.24 & 0.04 \\
& Generation cost & 1728.98 & 1727.69 & -1.28 & 1729.61 & 1719.88 & -9.73 \\
& Generation curtailment cost & 110.67 & 111.69 & 1.03 & 110.16 & 117.95 & 7.78 \\
& Load curtailment cost & 221.68 & 221.89 & 0.21 & 222.98 & 223.91 & 0.93 \\
\hline
\end{tabular}

By comparing Case I and Case II, the improvement in the profit in the proposed bidding strategy significantly increases as a higher penalty price factor is applied. Specifically, the improvement in the profit in the proposed bidding strategy increases by $54.8 \%$ more in Case II compared to Case I. This increase occurs because the imbalance cost of the basic and proposed bidding strategies change in different directions. Compared to the results of Case I, the imbalance cost of the basic bidding strategy in Case II increases from $\$ 54.04$ to $\$ 78.04$, but that of the proposed bidding strategy decreases from $\$ 13.10$ to $\$ 9.55$. Therefore, the difference in the imbalance cost between the two strategies in Case II has a larger value of $\$ 68.90$ than the $\$ 40.94$ in Case I. This is because the proposed bidding strategy can determine bids to avoid an excessive imbalance cost at the expense of the greater usage of the control capability of ANM, such as the output curtailment of RDG, the load curtailment, and the BESS. It can be deduced from the result in Table 5 that the differences in the generation curtailment cost, load curtailment cost, and battery wear cost are greater in Case II than in Case I. 


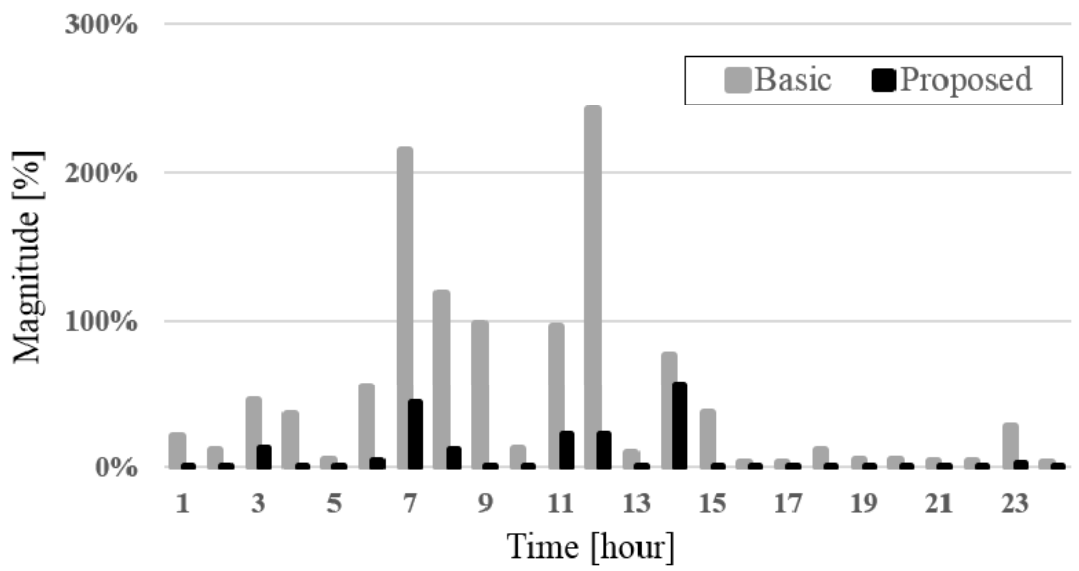

(a)

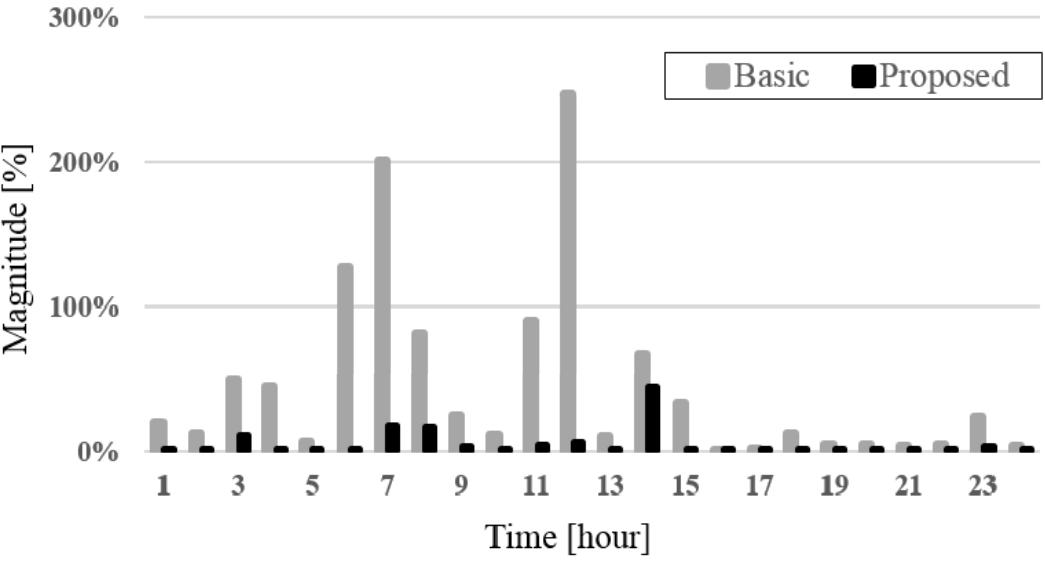

(b)

Figure 8. Magnitudes of the hourly expected imbalances normalized to the bids for each hour in the basic/proposed bidding strategies for (a) Case I and (b) Case II.

\section{Conclusions}

The performance of the proposed bidding strategy is verified through simulations with a renewable MG based on the IEEE 33-bus distribution network in [23]. The structure of the MG is shown in Figure 3. This study focuses on an optimal bidding strategy for a renewable MG with ANM. It is found that the proposed bidding strategy can significantly reduce the expected imbalance cost and increase the expected operating profit compared to the basic bidding strategy. This is because the proposed bidding strategy can determine day-ahead bids considering the change in the power flow on the connecting line by the real-time adjustments using ANM. Additionally, it is found that the improvement in the profit increases as the penalty price increases. Nevertheless, large expected imbalances still exist at some time stages in Figure 8, even if the proposed bidding strategy is applied. This imbalance comes from the large uncertainties associated with RDG and can harm the stability and reliability of the main grid. Therefore, it is necessary to develop the method for MGO to effectively manage this imbalance in real-time operation in further research.

Author Contributions: All the authors contributed to this work. Seung Wan Kim designed the study, performed the analysis, and wrote the first draft of the paper. Jip Kim set the simulation environment, performed simulations, and checked the overall logic of this work. Young Gyu Jin contributed to developing the mathematical model and thoroughly revised the paper. Yong Tae Yoon contributed to the conceptual approach and provided important comments on the modeling and analysis.

Conflicts of Interest: The authors declare no conflict of interest. 


\section{Nomenclatures}

The following nomenclatures are used in this manuscript:

- Abbreviations

$\begin{array}{ll}\text { MG } & \text { Microgrid } \\ \text { MGO } & \text { Microgrid Operator } \\ \text { SO } & \text { System Operator of the main grid } \\ \text { RDG } & \text { Renewable Distributed Generation } \\ \text { WT } & \text { Wind Turbine } \\ \text { PV } & \text { Photovoltaic system } \\ \text { BESS } & \text { Battery Energy Storage System } \\ \text { ANM } & \text { Active network management } \\ \text { MP } & \text { Market Price in day-ahead energy market } \\ \text { TOU } & \text { Time-of-Use tariff }\end{array}$

- Indices and Sets

$\begin{array}{ll}s & \text { Index of each bus in the } M G \\ i & \text { Index of each bus in the } M G \\ t & \text { Index of each time stage } \\ l & \text { Index of each distribution line } \\ T & \text { Set of time stages } \\ B & \text { Set of buses in the } M G\end{array}$

\section{- Functions}
$P R^{S}$
$R^{s}$
Daily operating profit for scenario $s$
$C_{L C U R T}^{S}$
Daily operating revenue for scenario $s$
$C_{G}^{s}$
Daily load curtailment cost for scenario $s$
$C_{G C U R T}^{s}$
Daily generation cost for scenario $s$
$C_{\text {LOSS }}^{S}$
$C_{E X}^{s}$
Daily generation curtailment cost for scenario $s$
$C_{I M B}^{s}$
Daily loss cost for scenario $s$
$C_{B M}^{s}$
Daily exchange cost in the market for scenario $s$
Daily imbalance cost for scenario $s$
$f_{\text {LOSS }, t}^{S}$
Daily battery wear cost for scenario $s$
Total loss in the $M G$ at time stage $t$ for scenario $s$

\section{- Variables}

$$
\begin{aligned}
& P_{L, i, t^{\prime}}^{s} Q_{L, i, t}^{s} \\
& P_{G, i, t^{\prime}}^{s} Q_{G, i, t}^{s} \\
& P_{B E S S, i, t^{\prime}}^{s} Q_{B E S S, i, t}^{s} \\
& S o C_{i, t}^{s} \\
& P_{C O N, t^{\prime}}^{s} Q_{C O N, t}^{s} \\
& P_{F, i, t^{\prime}}^{s} Q_{F, i, t}^{s} \\
& \left|V_{i, t}^{s}\right|, \delta_{i, t}^{s} \\
& P_{B I D, t}
\end{aligned}
$$

Dispatched active/reactive loads in the $i$-th bus at time stage $t$ for scenario $s$

Dispatched active/reactive power outputs of the RDG in the $i$-th bus at time stage $t$ for scenario $s$

Scheduled active/reactive powers of the BESS at the $i$-th bus at time stage $t$ for scenario $s$

State-of-charge of the BESS in the $i$-th bus at time stage $t$ for scenario $s$

Active/reactive power flows in the line connecting the main grid and $M G$ at time stage $t$ for scenario $s$

Active/reactive power flows in the $i$-th distribution line at time stage $t$ for scenario $s$

Magnitude/angle of the voltage of the $i$-th bus at time stage $t$ for scenario $s$

Submitted bid in the day-ahead energy market at time stage $t$ 


\section{- Parameters}

$\hat{P}_{D, i, t}^{s}, \hat{Q}_{D, i, t}^{s}$
$\hat{P}_{G, i, t}^{s}$
$P_{C A P, i}$
$S_{O} C_{t}^{M I N}, S_{O} C_{i}^{M A X}$
$P_{B E S S, i}^{M A X}$
$\bar{S}_{L I N E, i}, \bar{S}_{C O N}$
$p^{s}$
$\rho_{t}$
$\lambda_{t}$
$\mu_{U N D E R}, \mu_{O V E R}$
$\gamma_{L C U R T}, \gamma_{G C U R T}$
$b$
$\alpha_{B}, \beta_{B}$
$\Delta t$
$V_{L L}, V_{U L}$
$G_{i j}, B_{i j}$
$P F R{ }_{G}, P F R_{C O N}$

Forecasted active/reactive power demands in the $i$-th bus at time stage $t$ for scenario $s$

Forecasted active power output of the RDG in the $i$-th bus at time stage $t$ for scenario $s$

Capacity of the RDG in the $i$-th bus

Minimum/maximum state-of-charge of the BESS in the $i$-th bus at time stage $t$

Maximum power output of the BESS in the $i$-th bus

Capacities of the $i$-th distribution line and the line connecting the main grid and $M G$

Probability of scenario $s$

Retail price at time stage $t$

Day-ahead market price at time stage $t$

Penalty price factors for the under/over-scheduling imbalance Load and generation curtailment compensation factors

Half-width of the tolerance band

Fast charging and cycling cost parameters of the BESS

Duration of each time stage

Lower/upper voltage limits

Conductance/susceptance of the distribution line between the $i$-th bus and the $j$-th bus

Limit of the power factor for RDG and the power flow on the connecting line

\section{References}

1. Lasseter, R.; Akhil, A.; Marnay, C.; Stephens, J.; Dagle, J.; Guttromson, R.; Meliopoulous, A.S.; Yinger, R.; Eto, J. Integration of Distributed Energy Resources: The CERTS Microgrid Concept; Lawrence Berkeley National Laboratory: Berkeley, CA, USA, 2002.

2. Hatziargyriou, N.; Asano, H.; Iravani, R.; Marnay, C. Microgrids. IEEE Power Energy Mag. 2007, 5, 78-94. [CrossRef]

3. Hawkes, A.D.; Leach, M.A. Modelling high level system design and unit commitment for a microgrid. Appl. Energy 2009, 86, 1253-1265. [CrossRef]

4. Elsied, M.; Oukaour, A.; Gualous, H.; Hassan, R. Energy management and optimization in microgrid system based on green energy. Energy 2015, 84, 139-151. [CrossRef]

5. Lee, S.Y.; Jin, Y.G.; Yoon, Y.T. Determining the optimal reserve capacity in a microgrid with islanded operation. IEEE Trans. Power Syst. 2016. in press. [CrossRef]

6. Palizban, O.; Kauhaniemi, K.; Guerrero, J.M. Microgrids in active network management-Part I: Hierarchical control, energy storage, virtual power plants, and market participation. Renew. Sustain. Energy Rev. 2014, 36, 428-439. [CrossRef]

7. Niknam, T.; Golestaneh, F.; Malekpour, A. Probabilistic energy and operation management of a microgrid containing wind/photovoltaic/fuel cell generation and energy storage devices based on point estimate method and self-adaptive gravitational search algorithm. Energy 2012, 43, 427-437. [CrossRef]

8. Mohammadi, S.; Mozafari, B.; Solimani, S.; Niknam, T. An adaptive modified firefly optimization algorithm based on Hong's point estimate method to optimal operation management in a microgrid with consideration of uncertainties. Energy 2013, 51, 339-348. [CrossRef]

9. Mohammadi, S.; Soleymani, S.; Mozafari, B. Scenario-based stochastic operation management of microgrid including wind, photovoltaic, micro-turbine, fuel cell and energy storage devices. Int. J. Elect. Power Energy Syst. 2014, 54, 525-535. [CrossRef] 
10. Shi, L.; Luo, Y.; Tu, G.Y. Bidding strategy of microgrid with consideration of uncertainty for participating in power market. Int. J. Elect. Power Energy Syst. 2014, 59, 1-13. [CrossRef]

11. Nguyen, D.T.; Le, L.B. Risk-constrained profit maximization for microgrid aggregator with demand response. IEEE Trans. Smart Grid 2015, 6, 135-146. [CrossRef]

12. Zhang, J.; Fan, H.; Tang, W.; Wang, M.; Cheng, H.; Yao, L. Planning for distributed wind generation under active management mode. Int. J. Elect. Power Energy Syst. 2013, 47, 140-146. [CrossRef]

13. Carr, S.; Premier, G.C.; Guwy, A.J.; Dinsdale, R.M.; Maddy, J. Energy storage for active network management on electricity distribution networks with wind power. IET Renew. Power Gen. 2014, 8, 249-259. [CrossRef]

14. Gill, S.; Kockar, I.; Ault, G.W. Dynamic optimal power flow for active distribution networks. IEEE Trans. Power Syst. 2014, 29, 121-131. [CrossRef]

15. Yun, S.Y.; Chu, C.M.; Kwon, S.C.; Song, I.K.; Choi, J.H. The development and empirical evaluation of the Korean smart distribution management system. Energies 2014, 7, 1332-1362. [CrossRef]

16. Hernandez, L.; Baladron, C.; Aguiar, J.M.; Calavia, L.; Carro, B.; S-Esquevillas, A.; Sanjuan, J.; Gonzalez, A.; Lloret, J. Improved short-term load forecasting based on two-stage predictions with artificial neural networks in a microgrid environment. Energies 2013, 6, 4489-4507. [CrossRef]

17. Dragomir, O.E.; Dragomir, F.; Stefan, V.; Minca, E. Adaptive neuro-fuzzy inference systems as a strategy for predicting and controlling the energy produced from renewable sources. Energies 2015, 8, 13047-13061. [CrossRef]

18. Matevosyan, J.; Söder, L. Minimization of imbalance cost trading wind power on the short-term power market. IEEE Trans. Power Syst. 2006, 21, 1396-1404. [CrossRef]

19. California Independent System Operator (ISO). Business Practice Manual for the Energy Imbalance Market; California ISO: Folsom, CA, USA, 2014.

20. Shi, W.; Xie, X.; Chu, C.C.; Gadh, R. Distributed optimal energy management in microgrids. IEEE Trans. Smart Grid 2015, 6, 1137-1146. [CrossRef]

21. Safdarian, A.; Fotuhi-Firuzabad, M.; Lehtonen, M. A stochastic framework for short-term operation of a distribution company. IEEE Trans. Power Syst. 2013, 28, 4712-4721. [CrossRef]

22. Gabash, A.; Li, P. Active-reactive optimal power flow in distribution networks with embedded generation and battery storage. IEEE Trans. Power Syst. 2012, 27, 2026-2035. [CrossRef]

23. Baran, M.E.; Wu, F.F. Network reconfiguration in distribution systems for loss reduction and load balancing. IEEE Trans. Power Deliv. 1989, 4, 1401-1407. [CrossRef]

24. California ISO: Open Access Same-Time Information System. Available online: http:/ /oasis.caiso.com/ mrioasis/logon.do?tiny=X_JAPA (accessed on 6 November 2015).

25. Wang, J.; Shahidehpour, M.; Li, Z. Security-constrained unit commitment with volatile wind power generation. IEEE Trans. Power Syst. 2008, 23, 1319-1327. [CrossRef]

26. Bouffard, F.; Galiana, F.D. Stochastic security for operation planning with significant wind power generation. In Proceedings of the Power and Energy Society General Meeting-Conversion and Delivery of Electrical Energy in the 21st Century, Pittsburgh, PA, USA, 20-24 July 2008.

27. Pacific Gas and Electric Co: Electric Rates. Available online: http://www.pge.com/tariffs/electric.shtml (accessed on 6 November 2015).

(C) 2016 by the authors; licensee MDPI, Basel, Switzerland. This article is an open access article distributed under the terms and conditions of the Creative Commons by Attribution (CC-BY) license (http://creativecommons.org/licenses/by/4.0/). 